\title{
Neuroprotective effect of recombinant adeno-associated virus human thioredoxin-PR39 on acute cerebral infarction in rats
}

\author{
YU-DONG GUO ${ }^{1}$, TENG HUANG ${ }^{2}$, WEN-HUA SHENG ${ }^{3}$, YUN-FEI GUAN ${ }^{4}$, \\ YI-FENG DU ${ }^{5}$, YOU-TING LIN ${ }^{5}$ and XI-YUN RUAN ${ }^{5}$ \\ ${ }^{1}$ Department of Neurology, The Fifth People's Hospital of Jinan; ${ }^{2}$ Department of Neurology, \\ Shandong Provincial Hospital Affiliated to Shandong University (Western), Jinan, Shandong 250022; \\ ${ }^{3}$ Department of Neurology, Jinan Central Hospital Affiliated to Shandong University, Jinan, Shandong 250013; \\ ${ }^{4}$ Department of Neurology, School of Medicine, Shandong University, Jinan, Shandong 250012; \\ ${ }^{5}$ Department of Neurology, Shandong Provincial Hospital Affiliated to Shandong University, \\ Jinan, Shandong 250021, P.R. China
}

Received September 22, 2017; Accepted April 19, 2018

DOI: $10.3892 /$ etm.2018.6456

\begin{abstract}
The recombinant adeno-associated virus human thioredoxin-PR39(rAAV/hTRX-PR39) has been demonstrated to have a protective effect on hypoxic cells. The present study aimed to explore the potential effect of rAAV/hTRX-PR39 on acute cerebral infarction in rats. Middle cerebral artery occlusion (MCAO) model rats were produced and divided into three groups: Normal saline group, empty virus group (rAAV, without hTRX-PR39 cDNA) and rAAV/hTRX-PR39 group. Hematoxylin and eosin staining and electron microscopy observation were used to assess the morphological changes of ischemic brain tissue during different periods. Immunohistochemistry was employed to detect the expression of CD34 to reflect angiogenesis of ischemic brain tissue. Rats treated with $\mathrm{rAAV/hTRX}$-PR39 showed an alleviated degree of ischemic brain edema relative to that in control groups, suggesting PR39 can ameliorate brain damage after cerebral ischemia. In the rAAV/hTRX-PR39 group, CD34-positive cells were significantly increased in ischemic brain tissues compared to control groups. Furthermore, CD34-positive cells were primarily observed around the perivascular in ischemic brain, indicating the angiogenesis role of PR39 in ischemic brain. The present findings suggest that PR39 could effectively ameliorate ischemic brain damage and promote angiogenesis, which may contribute to the treatment of acute cerebral infarction.
\end{abstract}

Correspondence to: Professor Xi-Yun Ruan, Department of Neurology, Shandong Provincial Hospital Affiliated to Shandong University, 324 Jingwuweiqi Road, Huaiyin, Jinan, Shandong 250021, P.R. China

E-mail: xiyunruan@126.com

Key words: adeno-associated virus, thioredoxin, PR39, gene fusion, gene therapy, acute cerebral infarction

\section{Introduction}

Stroke is the second leading cause of death worldwide, of which over $80 \%$ cases are ischemic stroke caused by cerebral infarction (1). Approximately 6.9 million people had an ischemic stroke annually (2), and 3.3 million people died from this terrible disease (3). Although the stent retrieval technique has clear clinical benefits in many stroke patients, drug treatment is still the first option therapy for ischemic stroke (4). The drug recombinant tissue plasminogen activator (rt-PA) is the only FDA-approved medication for treating acute ischemic stroke (5), while several limitations hamper its utilization clinically (6). There is an unmet requirement for the development of effective drugs for stroke patients especially those who are inoperable.

As a new strategy with great potential applications, gene therapy provides promising approaches to the treatment of various diseases and has achieved good results $(7,8)$. A number of studies have indicated the protective roles of neurotrophic factors, antiapoptosis genes and angiogenic growth factors in brain infarction (9). Although great advances in gene therapy, the potential in treating disorders has fallen short of public expectations.

PR39 is a proline-arginine-rich peptide antibiotic with 39 amino acids (10). It has been confirmed that PR39, as the angiogenic Masterswitch protein, has a protective effect on myocardial ischemia-reperfusion injury by protective metabolic and survival responses through hypoxia inducible factor-1 (HIF1)- $\alpha$ stabilization (11). However, PR39 only may be ineffective for the treatment, because of the existence of blood-brain barrier. It is urgent to excavate an aptamer to improve the solubility and activity. Human thioredoxin (hTRX), as a natural human protein with low immunogenicity, has been used as a frame protein to construct a gene fusion system (12). hTRX is an ubiquitous, thiol-mediated protein that protects neurons against a variety of oxidative stresses and is considered as a promising target for clinical therapy $(13,14)$. It is indicated that hTRX gene fusion system 
could dramatically improve the activity of the expression products (12).

In our earlier study, we have successfully constructed recombinant adeno-associated virus human thioredoxin-PR39 (rAAV/hTRX-PR39), and confirmed its potential role in preventing cell apoptosis hypoxia-induced $(15,16)$. Moreover, transfection with rAAV/hTRX-PR39 in chicken embryo model was shown to promote angiogenesis and cell survival under hypoxic condition (17). In the present study, we aimed to explore the potential protective effects of hTRX-PR39 on acute cerebral infarction.

\section{Materials and methods}

Construction ofrecombinantvirus. ThepGEM-T-hTRX-PR39 vector containing hTRX-PR39 full-length gene sequence was constructed as previously described (15). We first generated PR39 cDNA including EcoR721 and BamHI restriction enzyme sites and hTRX cDNA including EcoR721 and $E c o$ RI restriction enzyme sites. Then, the synthesized fragments were cloned into a pGEM-T vector, respectively. The positive clone was identified using restriction enzymes, and the cloned amplified fragments were sequenced by the dideoxy-mediated chain-termination method. Subsequently, pGEM-T-hTRX and pGEM-T-PR39 were digested by BamHI and EcoRI, and recombined a vector. Finally, the recombinant vector pGEM-T-hTRX-PR39 was produced. The viral vector, plasmid and corresponding cell lines were all purchased from Xi'an Huaguang Biological Engineering Co., Ltd. (Xi'an, China).

Animal model and experimental protocol. In this study, 20 female Sprague-Dawley rats weighting 280-300 g were purchased from Shanghai Slack Laboratory Animal Co., Ltd. (Shanghai, China; license no. SCXK(HU)2012-0002). All experiments were approved by the Shandong University Institutional Animal Care and Use Committee.

Middle cerebral artery occlusion (MCAO) was produced using the Longa suture-occluded technique (18). The neurological evaluation was performed $2 \mathrm{~h}$ after MCAO with Bederson's test (19). Rats were tail suspended. Then they showed typical signs and symptoms, such as buckling and elevating of the contralateral forelimb, shoulder adduction and elbow extending.

According to random number table method, experimental rats were divided into three groups: Normal saline group (Control 1 group, $\mathrm{n}=4$ ), empty virus group (Control 2 group, $\mathrm{n}=4$ ) and rAAV/hTRX-PR39 group (PR39 group, n=12). The catheter was inserted into the middle cerebral artery via the rat intravenous administration. Rats were injected with isovolumetric physiological saline, isovolumetric empty virus fluid and $3 \times 10^{9}$ pfu rAAV-hTRX-PR39 fluid, respectively.

Hematoxylin and eosin staining $(H \& E)$. Three experimental rats in PR39 group and one rat in each control group were sacrificed at time points of $12 \mathrm{~h}, 1,2$ and 3 weeks after injection. The rat brains were fixed in formalin and embedded in paraffin. The ischemic brain tissues were sectioned into equally spaced $(2 \mathrm{~mm})$ coronal blocks. A series of adjacent $4 \mu \mathrm{m}$-thick slices were cut from each block. Then, the tissue sections were counterstained with $\mathrm{H} \& \mathrm{E}$ for histologic examination. H\&E staining results of ischemic tissues were observed and imaged by microscope (BH2; Olympus, Tokyo, Japan). Six positive visions (magnification, $\mathrm{x} 400$ ) of each brain slice were randomly selected for $\mathrm{CD} 34$ positive cells counting.

Immunohistochemistry test. The cerebral tissues were cut into 4 equally spaced $(2 \mathrm{~mm})$ coronal blocks. Immunohistochemistry test was performed with well-characterized mouse monoclonal antibody against CD34 (1:200; Proteintech Group Inc., Wuhan, China). Brain sections ( $4 \mu \mathrm{m}$-thick) were fixed in $4 \%$ paraformaldehyde for $30 \mathrm{~min}$ at $37^{\circ} \mathrm{C}$. Then, the sections were incubated with primary antibody at $4^{\circ} \mathrm{C}$ overnight followed by biotinylated secondary antibody (1:200; Proteintech Group Inc.) for $2 \mathrm{~h}$ at $37^{\circ} \mathrm{C}$. Subsequently, sections were incubated with avidin-biotin complex labeled with alkaline phosphatase (AP), which then reacted with diaminobenzidine, for $60 \mathrm{~min}$ at $37^{\circ} \mathrm{C}$. Omitting primary antibody was considered as a negative control.

Electron microscopy observation. Cerebral slices were fixed in $2.5 \%(\mathrm{v} / \mathrm{v})$ glutaraldehyde for $2 \mathrm{~h}$ and $1 \%(\mathrm{v} / \mathrm{v})$ osmic acid for 2-3 $\mathrm{h}$, followed by dehydration in a graded series of ethanol and $90 \%(\mathrm{v} / \mathrm{v})$ acetone for 15-20 min. After embedding and solidifying it in an epoxy resin, ultra-thin slices were cut at $50-60 \mathrm{~nm}$ and stained with 3\% (w/v) uranyl acetate and lead citrate. Electron microscopic images were ere observed and photographed under a transmission electron microscopy (TECNAI 10; Philips, Eindhoven, Netherlands).

Statistical analysis. Data are expressed as the mean \pm standard deviation. The two-sample t-test was used for comparisons between groups. Statistical analysis was carried out by SPSS software (SPSS version 13.0; SPSS, Inc., Chicago, IL, USA). $\mathrm{P}<0.05$ was considered to indicate a statistically significant difference.

\section{Results}

PR39 ameliorates brain damage after cerebral ischemia. By $\mathrm{H} \& \mathrm{E}$ staining, brain lesion was observed in the ischemic core of the striatum and cortex in the rats subjected to MCAO. In two control groups, brain tissues showed gradually growing cell edema and angioedema over time. At the time points of $12 \mathrm{~h}$ and 1 week, brain tissue in all three groups showed same lesions after MCAO treatment. However, the degree of ischemic brain edema in the rats treated with rAAV/hTRX-PR39 was alleviated relative to that in control groups at the time points of 2 and 3 weeks. At $12 \mathrm{~h}$ after treatment, brain tissue in all three groups showed slight lesions. There was no cell degeneration and necrosis. At 1 week after treatment, the size of nerve cells was still consistent. Nucleus staining was uniform. While the gap around nerve cells and blood vessels widened slightly, indicating mild cell edema and angioedema. At 2 and 3 weeks after treatment, the degree of tissue edema was aggravated in two control groups, and the edema appeared in the cerebral medulla and white matter. While in PR39 group, brain edema was alleviated and nerve cells showed no obvious morphological degeneration and necrosis. The results were shown in Fig. 1. 


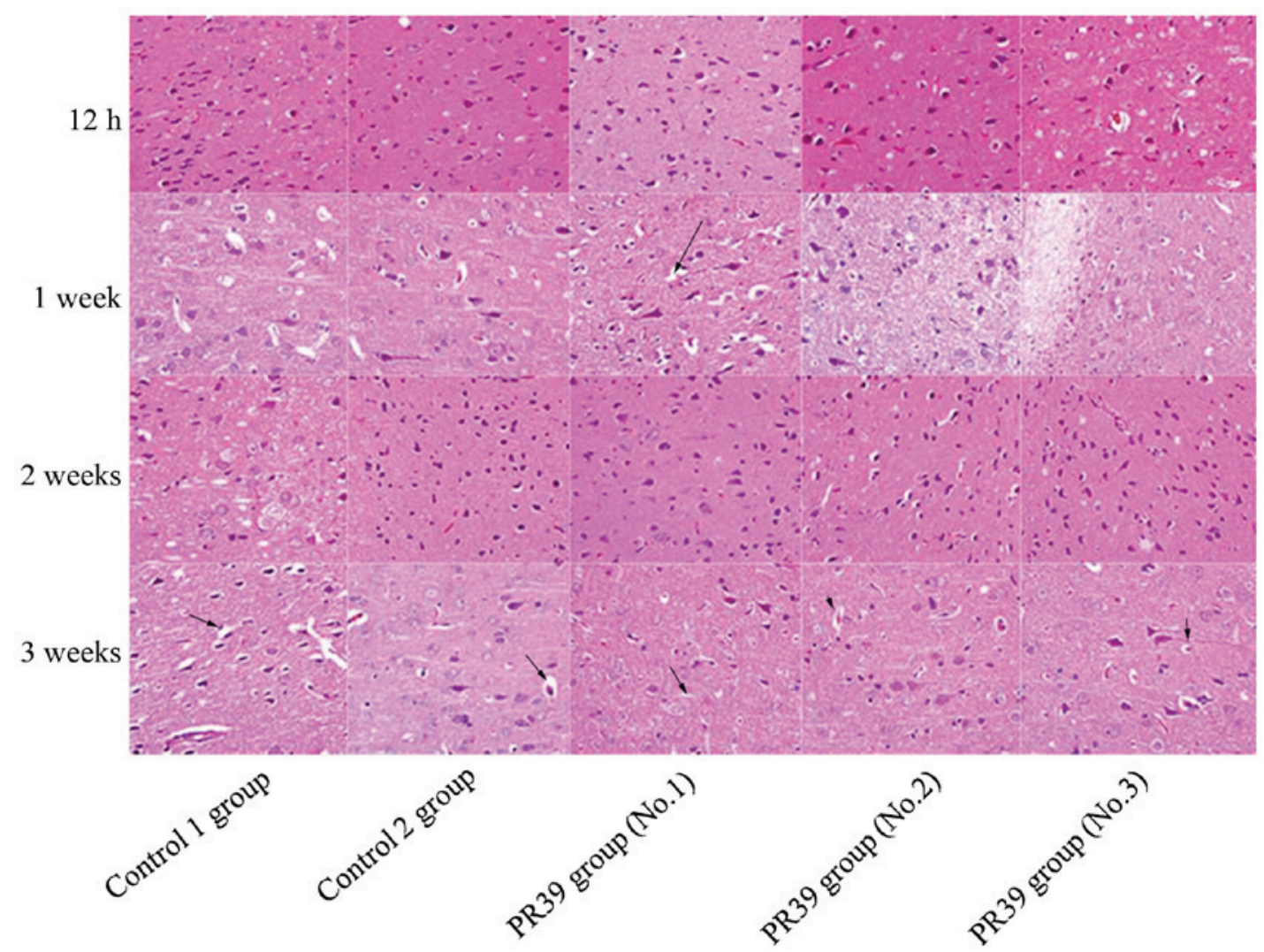

Figure 1. Hematoxylin and eosin staining results of ischemic brain sections. The gap around nerve cells and blood vessels indicated the cell edema and angioedema, which was labeled by black arrows (magnification, x400).

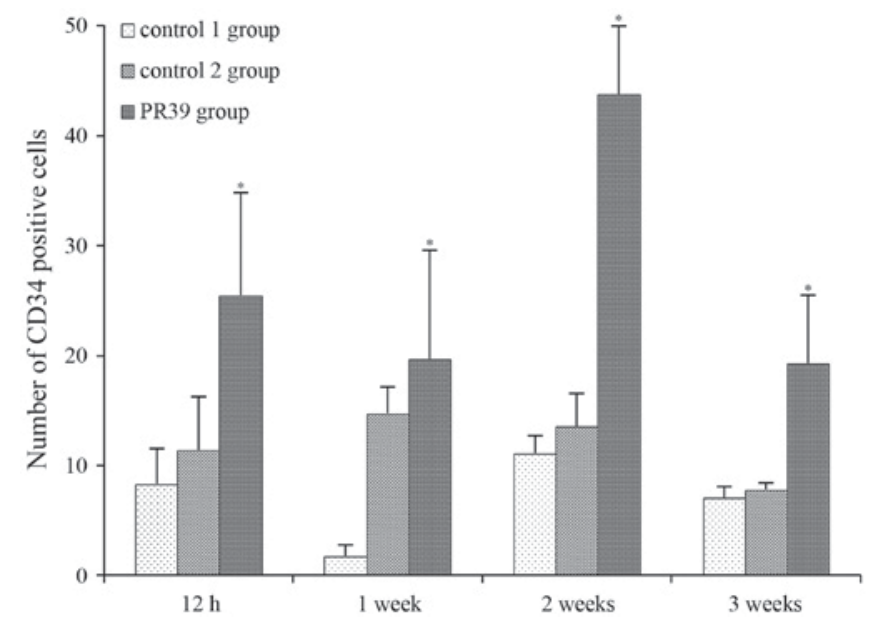

Figure 2. Histogram showing number of CD34-positive cells in ischemic brain sections. Immunostaining analysis showed that the number of CD34-positive cells increased significantly in PR39 treated group compared with control groups. "P<0.05.

PR39 promotes CD34-positive cells in ischemic brain. Immunostaining analysis showed that PR39 significantly increased the number of CD34 immunoreactive cells in ischemic brain tissues at $12 \mathrm{~h}, 1,2$ and 3 weeks after MCAO compared to saline control group and empty virus group $(\mathrm{P}<0.05)$, as shown in Fig. 2. CD34-positive cells were mainly seen around the perivascular in ischemic brain. The cytoplasm of CD34-positive cells was brown, which had a sharp contrast with negative cells (Fig. 3).
Electron microscopy observation. Ultrastructure of cerebral ultra-thin slices was examined with electron microscopy (Fig. 4). The neurons structure changes were found in two control groups (normal saline group and empty virus group). Electron microscopy showed apoptotic neurons in control groups and the cell morphology was characterized by irregular and shriveled nucleus and disruption of nuclei membrane integrity. The cell morphological structures were similar in normal saline group and empty virus group, such as mitochondrial swelling and vacuolation. While, cells treated with PR39 exhibited integrated nucleus membrane and higher electron density of mitochondria. The vascular structure changes were also found under electron microscopy in two control groups, such as the vascular endothelial cell swelling and cavitation formation. In PR39 group, the structure of vascular endothelial cells was more complete relative to control groups.

\section{Discussion}

This study investigated the neuroprotective effect of hTRX-PR39 on acute cerebral infarction. Morphological changes of ischemic brain tissues showed that the damage degree of ischemic brain in rats treated with rAAV/hTRX-PR39 was slighter than that of rats in control groups at 2 and 3 weeks. Moreover, immunohistochemical method found that rAAV/hTRX-PR39 could promote angiogenesis by detecting the expression of CD34, the most sensitive endothelial marker with the highest antigen specificity in nascent microvessels. Our results showed that rAAV/hTRX-PR39 treatment could successfully reduce damage of ischemic brain tissues and promote angiogenesis in MCAO rats. 


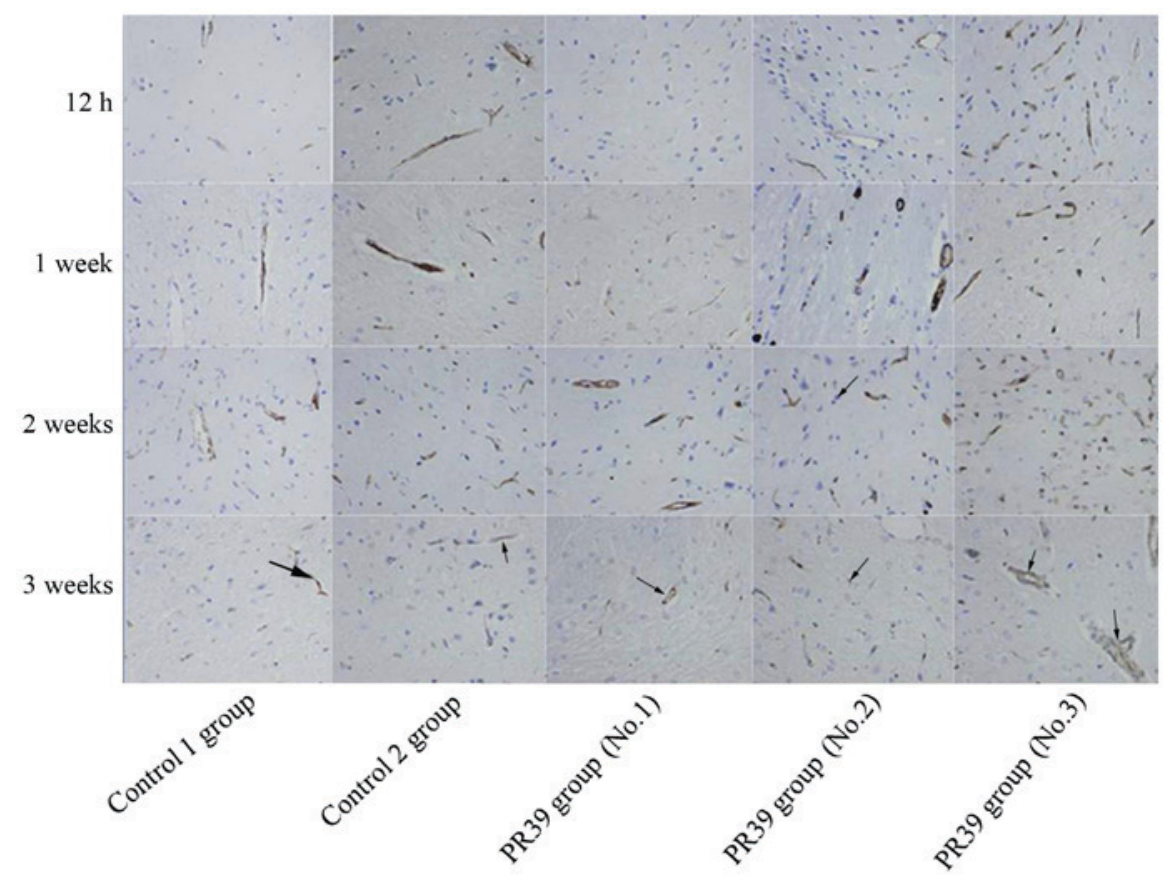

Figure 3. Immunohistochemistry results of ischemic brain sections. PR39 treatment induced an increase in CD34 immunoreactive cells after middle cerebral artery occlusion. CD34-positive expression was mainly found in the blood vessel walls. The cytoplasm of CD34-positive cells was stained brown (indicated by the black arrows). Magnification, $\mathrm{x} 400$.

$12 \mathrm{~h}$
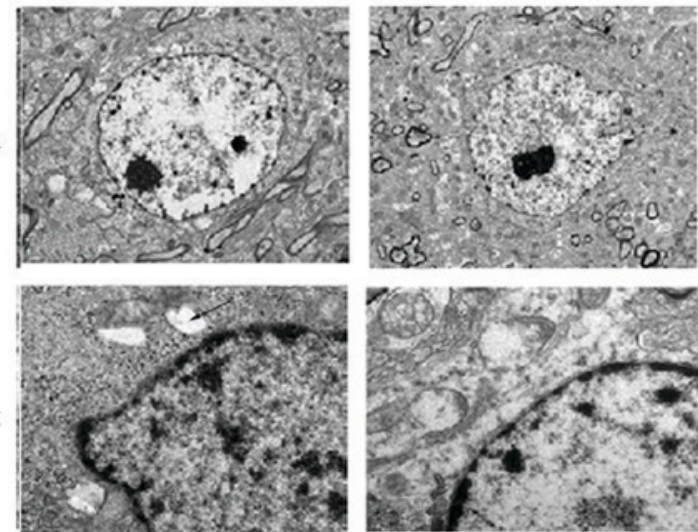

1 week
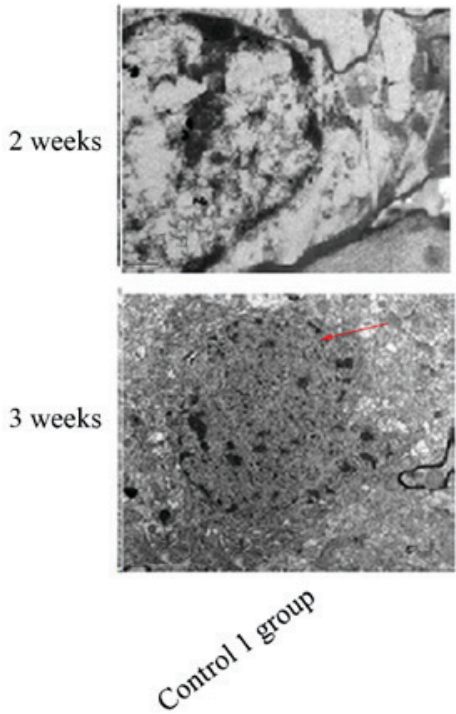
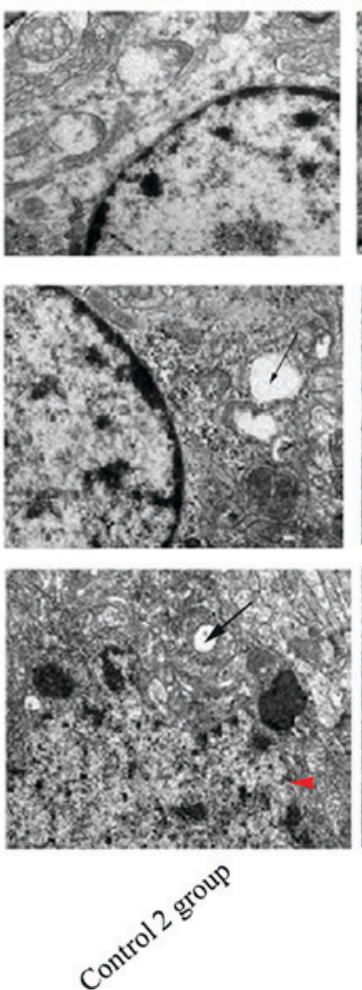
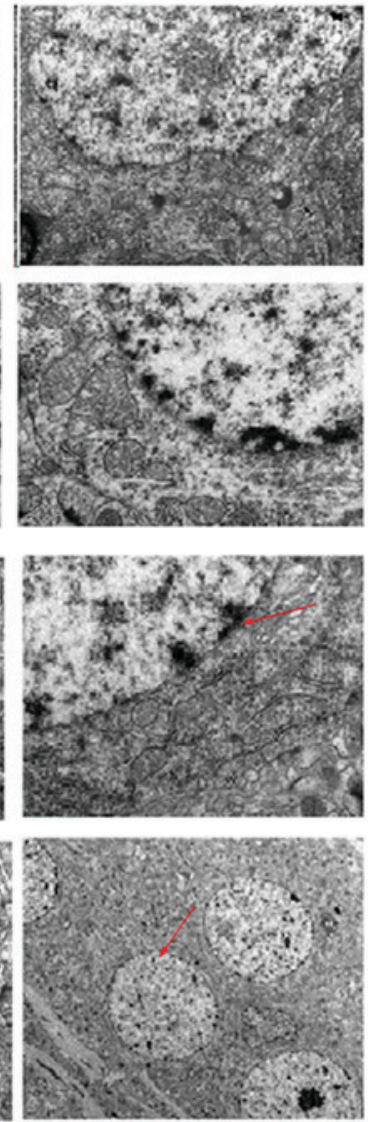

$83^{39^{300^{009}}}$

Figure 4. Electron microscopy results showing ultrastructure of cerebral ultra-thin slices. Electron microscopy demonstrated apoptotic neurons in control groups and integrated nucleus membrane in PR39 group. In PR39 group, the structure of vascular endothelial cells was more complete relative to control groups. Mitochondrial vacuolation was labeled by black arrows, while nucleus membrane was labeled by red arrows. 
In biology, PR39 has multiple biological functions, such as anti-inflammatory, immunoregulation, stimulating angiogenesis and apoptosis inhibition (20). Wu et al (21) confirmed the antiapoptotic role in hypoxic endothelial cells by blocking apoptosis signal transduction pathway. It can quickly pass through the endothelial cell membrane by specific binding to SH3 domain structure $\left[\mathrm{p} 130^{\mathrm{Cas}}(22)\right.$ and $\left.\mathrm{p} 47^{\mathrm{phox}}(23)\right]$. Moreover, previous study indicated that PR39 can decrease the ischemic-reperfusion injury by inhibiting the degradation of $\mathrm{I} \kappa \mathrm{B} \alpha$ and adhesion molecule (24). In addition, PR39 can block the degradation of HIF1- $\alpha$ and improve the expression of multiple angiogenic proteins, such as VEGF, KDR and FLT-1 (17,25-27), illustrating the crucial role of PR39 in confronting ischemic damage.

To solve the problem of repeating dosing, rAAV vector was employed as a carrier for gene transfering. It is indicated that rAAV shows a series of advantages, such as low immunogenicity, high transfection efficiency, targeted integration and long-term stability of gene expression (28). A great number of studies have stated that rAAV vector is a safe and suitable tool for gene therapy in ischemic stroke $(29,30)$. So far, rAAV has become the most common gene vector for the central nervous system disorder. To improve the activity of the expression products and increase the solubility of PR39 to pass blood-brain barrier, hTRX was utilized to construct the gene fusion system in our study. Moreover, hTRX could inhibit thrombosis by reducing tissue factor activity and further prevent the injury of the ischemic tissue (31). Consistent with An et al report, intramyocardial injection of recombinant adeno-associated viral vector coexpressing pr39/adrenomedullin enhances angiogenesis and reduces apoptosis in a rat myocardial infarction model (32). Our study showed that the gene fusion system rAAV/hTRX-PR39 can effectively ameliorate acute cerebral infarction-induced ischemic injury by promoting angiogenesis and inhibiting cell apoptosis. As a limitation in this report, the intracranial transfection and expression of rAAV/hTRX-PR39 and rat behavior or brain MR tests have not been performed, which will be added to the future report.

In conclusion, in this study, rAAV/hTRX-PR39 successfully reduced ischemic brain damage and promoted angiogenesis, suggesting the underlying application in the treatment of acute cerebral infarction.

\section{Acknowledgements}

The study was supported by the National Natural Science Foundation of China (grand no. 30970992).

\section{References}

1. O'Donnell MJ, XavierD,Liu L, Zhang H, Chin SL, Rao-MelaciniP, Rangarajan S, Islam S, Pais P, McQueen MJ, et al: Risk factors for ischaemic and intracerebral haemorrhagic stroke in 22 countries (the INTERSTROKE study): A case-control study. Lancet 376: $112-123,2010$.

2. Global Burden of Disease Study 2013 Collaborators: Global, regional, and national incidence, prevalence, and years lived with disability for 301 acute and chronic diseases and injuries in 188 countries, 1990-2013: A systematic analysis for the Global Burden of Disease Study 2013. Lancet 386: 743-800, 2015.

3. GBD 2013 Mortality and Causes of Death Collaborators: Global, regional, and national age-sex specific all-cause and cause-specific mortality for 240 causes of death, 1990-2013: A systematic analysis for the Global Burden of Disease Study 2013. Lancet 385: 117-171, 2015.
4. Walcott BP, Boehm KM, Stapleton CJ, Mehta BP, Nahed BV and Ogilvy CS: Retrievable stent thrombectomy in the treatment of acute ischemic stroke: Analysis of a revolutionizing treatment technique. J Clin Neurosci 20: 1346-1349, 2013.

5. Brainin M, Teuschl Y and Kalra L: Acute treatment and long-term management of stroke in developing countries. Lancet Neurol 6: 553-561, 2007.

6. Wang W, Li M, Chen Q and Wang J: Hemorrhagic transformation after tissue plasminogen activator reperfusion therapy for ischemic stroke: Mechanisms, models, and biomarkers. Mol Neurobiol 52: 1572-1579, 2015.

7. Ooboshi H, Ibayashi S, Takada J, Kumai Y and Iida M: Brain ischemia as a potential target of gene therapy. Exp Gerontol 38: 183-187, 2003.

8. Moss JA: Gene therapy review. Radiol Technol 86: 155-184, 2014

9. Aoki M and Morishita R: Therapeutic angiogenesis for ischemic diseases. Nihon Rinsho 64: 762-768, 2006 (In Japanese).

10. Gudmundsson GH, Magnusson KP, Chowdhary BP, Johansson M, Andersson L and Boman HG: Structure of the gene for porcine peptide antibiotic PR-39, a cathelin gene family member: comparative mapping of the locus for the human peptide antibiotic FALL-39. Proc Natl Acad Sci USA 92: 7085-7089, 1995.

11. Muinck ED, Nagy N, Tirziu D, Murakami M, Gurusamy N, Goswami SK, Ghatpande S, Engelman RM, Simons M and Das DK: Protection against myocardial ischemia-reperfusion injury by the angiogenic Masterswitch protein PR 39 gene therapy: the roles of HIF1alpha stabilization and FGFR1 signaling. Antioxid Redox Signal 9: 437-445, 2007.

12. Borghouts C, Kunz C, Delis N and Groner B: Monomeric recombinant peptide aptamers are required for efficient intracellular uptake and target inhibition. Mol Cancer Res 6: 267-281, 2008.

13. Yamawaki H, Haendeler J and Berk BC: Thioredoxin: A key regulator of cardiovascular homeostasis. Circ Res 93: 1029-1033, 2003.

14. Yoshida T, Nakamura H, Masutani $\mathrm{H}$ and Yodoi J: The involvement of thioredoxin and thioredoxin binding protein-2 on cellular proliferation and aging process. Ann N Y Acad Sci 1055: 1-12, 2005.

15. Ruan XY, Bi JZ, Liu QY, Zhang SB, Yang GX and Wang QY: Construction and identification of recombinant plasmids expressing hTRX-PR39. J Shandong University (Health Sciences) 47: 30-34, 2009.

16. Ruan X, Yuan Z, Du Y, Yang G and Wang Q: Recombinant adeno-associated virus delivered human thioredoxin-PR39 prevents hypoxia-induced apoptosis of ECV304 cells. Neural Regen Res 7: 708-713, 2012.

17. Ruan XY, Liang YC, Du B, Lin YT, Guo YD, Zhao J, Li S, Li JF, Sun QJ and Du YF: Potential role of recombinant adeno-associated virus human thioredoxin-PR39 in cell and vascular protection against hypoxia. Exp Ther Med 9: 1605-1610, 2015.

18. Longa EZ, Weinstein PR, Carlson S and Cummins R: Reversible middle cerebral artery occlusion without craniectomy in rats. Stroke 20: 84-91, 1989.

19. Bederson JB, Pitts LH, Tsuji M, Nishimura MC, Davis RL and Bartkowski H: Rat middle cerebral artery occlusion: Evaluation of the model and development of a neurologic examination. Stroke 17: 472-476, 1986.

20. Ross CR, Ricevuti G and Scovassi AI: The antimicrobial peptide PR-39 has a protective effect against HeLa cell apoptosis. Chem Biol Drug Des 70: 154-157, 2007.

21. Wu J, Parungo C, Wu G, Kang PM, Laham RJ, Sellke FW, Simons M and Li J: PR39 inhibits apoptosis in hypoxic endothelial cells: Role of inhibitor apoptosis protein-2. Circulation 109: 1660-1667, 2004.

22. Chan YR and Gallo RL: PR-39, a syndecan-inducing antimicrobial peptide, binds and affects p130(Cas). J Biol Chem 273: 28978-28985, 1998.

23. Shi J, Ross CR, Leto TL and Blecha F: PR-39, a proline-rich antibacterial peptide that inhibits phagocyte NADPH oxidase activity by binding to Src homology 3 domains of 447 phox. Proc Natl Acad Sci USA 93: 6014-6018, 1996.

24. Bao J, Sato K, Li M, Gao Y, Abid R, Aird W, Simons M and Post MJ: PR-39 and PR-11 peptides inhibit ischemia-reperfusion injury by blocking proteasome-mediated I kappa B alpha degradation. Am J Physiol Heart Circ Physiol 281: H2612-H2618, 2001.

25. Li J, Post M, Volk R, Gao Y, Li M, Metais C, Sato K, Tsai J, Aird W, Rosenberg RD, et al: PR39, a peptide regulator of angiogenesis. Nat Med 6: 49-55, 2000 . 
26. Carmeliet P, Dor Y, Herbert JM, Fukumura D, Brusselmans K, Dewerchin M, Neeman M, Bono $F$, Abramovitch R, Maxwell P, et al: Role of HIF-1alpha in hypoxia-mediated apoptosis, cell proliferation and tumour angiogenesis. Nature 394 485-490, 1998

27. Sun L, Hao Y, Nie X, Xu J, Li Z, Zhang W, Liu Y and Zhang X: Recombinant AAV-PR39-mediated hypoxia-inducible factor $1 \alpha$ gene expression attenuates myocardial infarction. Int $\mathrm{J} \mathrm{Mo}$ Med 33: 171-177, 2014.

28. Weinberg MS, Samulski RJ and McCown TJ: Adeno-associated virus (AAV) gene therapy for neurological disease. Neuropharmacology 69: 82-88, 2013.

29. Li ZJ and Wang RZ: rAAV vector-mediated gene therapy for experimental ischemic stroke. Neurol India 56: 116-121, 2008.
30. Chtarto A, Bockstael O, Tshibangu T, Dewitte O, Levivier M and Tenenbaum L: A next step in adeno-associated virus-mediated gene therapy for neurological diseases: Regulation and targeting. Br J Clin Pharmacol 76: 217-232, 2013.

31. Wang P, Wu Y, Li X, Ma X and Zhong L: Thioredoxin and thioredoxin reductase control tissue factor activity by thiol redox-dependent mechanism. J Biol Chem 288: 3346-3358, 2013.

32. An R, Xi C, Xu J, Liu Y, Zhang S, Wang Y, Hao Y and Sun L: Intramyocardial injection of recombinant adeno-associated viral vector coexpressing PR39/adrenomedullin enhances angiogenesis and reduces apoptosis in a rat myocardial infarction model. Oxid Med Cell Longev 2017: 1271670, 2017. 